23 Eminson D, Postlethwaite R. Factitious illness: recognition and management. Arch Dis Child 1992;67:1510-6.

24 Sigal M, Gelkopf $M$, Meadow R. Munchausen by proxy syndrome: the triad of abuse, self-abuse, and deception. Compr Psychiatry 1989;30:527-33.

25 Department of Health and Social Security. Report of the committee of inquiry into the care and supervision provided in relation to Maria Colvell. London: HMSO, 1974:61-76.

26 Sugar J, Belfer M, Israel E, Herzog D. A 3-year-old boy's chronic diarrhea and unexplained death (clinical conference). f Am Acad Child Psychiatry 1991:30:1015-21.
27 Former nurse committed for trial accused of murdering four children and attempting to murder another nine. Times 1992;March 12:20.5b.

28 Rosenberg D. Web of deceit: a literature review of Munchausen syndrome by proxy. Child Abuse Negl 1987;11:547-63.

29 Bhugra D. Psychiatric Munchausen's syndrome. Acta Psychiatr Scand 1988;77:497-503.

30 Asher R. Munchausen syndrome. Lancet 1951;i:339-41.

\title{
Ethical use of covert videoing for potentially life threatening child abuse: a response to Drs Foreman and Farsides
}

\author{
David P Southall, Martin P Samuels
}

Foreman and Farsides suggest that the Children Act 1989 provides sufficient ways of protecting children from abuse without the need for covert video surveillance. To our knowledge, the intentional suffocation and poisoning of children by parents are the only forms of child abuse in which covert videoing has been reported. ${ }^{1-3}$ We will focus on its use in suffocation.

Intentional suffocation presents as recurrent cyanotic-apnoeic episodes of unknown cause in infants and young children. Such events can result in recurrent loss of consciousness, cerebral injury, lung injury (personal observations), or sudden death. ${ }^{4}$ Foreman and Farsides argue that covert videoing may be an inappropriate investigation for such events because the technique is incompatible with the Children Act; it sets the child up for further abuse in order to make the diagnosis; it involves a breach of confidentiality and trust between the paediatrician, social worker, nurses, and parent; and it can be replaced by an assessment order with overt video surveillance.

\section{Validity under Children Act}

We do not agree that covert videoing is incompatible with the Children Act, which states as its primary objective the welfare of the child. Although the argument about balance of probability within the act is clear, it is incumbent on all agencies working together on cases of suspected suffocation to obtain the most objective and definitive information to ensure the best protection of the child. Uncertainty arising from insufficient information could lead to sudden death, cerebral injury, or long term hidden emotional and physical torment that the child and his or her siblings have little or no defence against. ${ }^{5}$

Foreman and Farsides argue that involvement of doctors rather than the local authority in diagnosing this form of abuse is not always appropriate and could be harmful. We agree with this and consider that all groups must work together. Before starting covert videoing, a multidisciplinary strategy meeting should be convened by a senior social worker to consider the information that exists about the child and family. At this meeting paediatric medical and nursing staff, social workers, police officers, and legal representatives can discuss whether there is sufficient "information to act on" concerning the diagnosis of suffocation. Covert videoing would be used only where it is felt that the diagnosis is sufficiently uncertain and the child will not be protected in the long run.

The courts generally welcome the evidence obtained by covert videoing as it is usually definitive and cases are uncontested. This thereby reserves limited resources, both in time and in funding, for other children requiring help under the Children Act (one of its main principles is to avoid delay).

\section{The need for further abuse to make the diagnosis}

Although covert videoing requires further abuse to establish the diagnosis, this abuse is taking place in hospital under continuous surveillance and should be intercepted early. Observers can alert nursing staff to intervene as soon as suffocation has begun (usually a maximum of 25 seconds). ${ }^{6}$ Loss of consciousness occurs after 60-80 seconds and episodes of suffocation at home may last for 2-4 minutes (based on physiological recordings). Thus the risk of an "observed" episode is almost certainly less than that of returning a child to a parent because of insufficient information about the cause of the cyanotic-apnoeic events.

Provided that appropriate guidelines and standards are established, it should be possible to minimise the risk during covert videoing. This can be achieved by establishing a protocol in conjunction with the social services department, the police, and the area child protection team. Such a protocol should ensure that the child is in constant view; that a foolproof system exists for communicating between observers and the ward nurse responsible for intervention; that the child's oxygenation is monitored and alarms can be heard by the observers; and that observers are properly trained, with instruction in the use of video technology, a review of previously recorded events, and one to one discussion with the paediatrician in charge.

Other unexpected forms of abuse with immediately damaging effects, such as fractures, may not be (and have not been) prevented by the above guidelines. Without the information that covert videoing provides, however, there may be insufficient information to protect the child in the long term, with the result that such injuries happen later at home. Nevertheless, every attempt must be made to minimise the potential for further abuse during covert videoing.

\section{Breach of trust}

The breach of trust between paediatrician, social worker, nurse, and parent is regrettable but, in our view, if a parent may be inflicting life threatening abuse on his or her child this becomes justifiable. ${ }^{7}$ The professional's duty is to the child first and to the parents second, recognising that the child lives within the family.

Any protocol that uses covert videoing must ensure that it is the child and not the parent who is under observation at all times. As suffocation is generally a problem in infants and preschool children they are unlikely to suffer embarrassment. Foreman and Farsides raised the potential for problems associated with a failure to detect abuse with covert videoing. This is uncommon: 3 out of 28 cases in one centre's experience. In one of the three cases abnormal interactions between the parent and child were observed whenever the parent thought she was alone.
Correspondence to:

BMY 1993;307:613-4 


\section{Use of an assessment order}

Foreman and Farsides propose separating the child from the suspected perpetrator under an assessment order perhaps with overt video surveillance to document any cyanotic-apnoeic events.

Assessment orders are made "where significant harm is suspected but the child is not thought to be at immediate risk." Such an order is therefore inappropriate in the case of severe cyanotic-apnoeic episodes that may be due to intentional suffocation because the child is at immediate risk of sudden death

The fact that no episodes occurred during overt surveillance would, in Foreman and Farsides' opinion, represent an important piece of information and suggest that the parent was responsible for the events. This has two main problems. Firstly, if the hypoxaemic events had natural causes (and this is often impossible to exclude) the child may have a potentially life threatening event under less than optimal monitoring. During covert videoing there are usually two observers watching the child and listening to the output from the clinical monitors. Overt surveillance would be safe only with one to one specialist nursing and, if abuse is the mechanism, such nursing could go on for a long time. Conversely, the median duration of covert surveillance before abuse was detected was 24 hours in one study. ${ }^{6}$

Secondly, recurrent cyanotic-apnoeic episodes vary in their frequency and tend to cluster even when they occur naturally. They can also resolve as the child gets older. Such arguments have been used by paediatricians who have defended parents in cases of suspected suffocation where covert videoing has not been used. Alarms on clinical monitors can go off without a medical problem having occurred (false alarms), suggesting to observers that, despite separation of the parent and child, a natural medical problem is present. This argument is difficult to counter unless simultaneous physiological recordings are made.

Another problem that can arise during open assessment is the occurrence of a normal cyanotic or apnoeic episode. Between $1 \%$ and $3 \%$ of all infants and young children hold their breath and go blue when they cry. During sleep normal infants and children may stop breathing for up to 20 seconds. If these normal phenomena occur when a child is being monitored under an assessment order, it may be wrongly assumed that all the hypoxaemic episodes occurred naturally.

\section{Other points of contention}

The concept that parents will admit to inducing illness in their child provided that they are approached in a sympathetic manner by an expert counsellor, psychiatrist, or paediatrician has been discredited. ${ }^{6}$ Although a combination of separation and confrontation can be used to diagnose Munchausen syndrome by proxy when there is continuous abuse such as poisoning or the withholding of food, we believe that it is unsafe to use these methods for suffocation, which is discontinuous yet immediately life threatening. When parents have been confronted after video evidence has been collected, but before this has been revealed, they invariably deny their involvement. ${ }^{6}$ Such parents have plausible arguments and lie convincingly. Unfortunately the process of confrontation will almost always prevent definitive evidence being subsequently obtained with covert videoing.
Circumstantial data concerning parental ability, previous history of personality problems and psychiatric disturbance, a history of previous child abuse, etc, are important evidence to support the concept that the parent is abusing the child. However, such evidence may not be sufficiently powerful to result in long term protection. Although interim care orders can be obtained in the short to medium term, defending barristers and expert witnesses can often persuade the court to give a parent the benefit of the doubt when there is a lack of definite evidence (covert videoing). ${ }^{8}$

The main advantage of covert videoing is that it aims not only for the temporary protection of the child but for long term protection, continuing until the abusing parent is no longer a risk to this child or any future children. One problem with the Children Act is that although it aims to prevent serious harm, it does not define the duration of this protection. Parents who suffocate their children usually have a personality disorder. This may be extremely difficult to treat and will certainly not resolve over the course of a month or two, if ever.

Examination of covert video recordings of children who are being suffocated shows that some are subjected to other forms of serious abuse. Children have been verbally and physically abused in less severe but still harmful ways during short periods of observation (2-3 hours) before more definitive abuse. The long term suffering endured by such children at home is difficult to imagine. We believe that it is the long term protection of the child that is most important and, given the available data, we reject the ethics of trying out the new assessment order approach and reverting to covert videoing if it proves significantly flawed.

The statement of Foreman and Farsides that covert videoing should not be used solely to detect crime belittles the work of the police, who play an extremely important part in protecting children. Repeated suffocation is a serious crime against a child and should be identified with all possible care so that it can be permanently prevented.

Intentional suffocation, presenting as recurrent apnoeic-cyanotic episodes and with features of Munchausen syndrome by proxy, overlaps with other severe forms of chlld abuse. Rather than arguing over the ethics of covert videoing, we should perhaps be asking a more fundamental question about why such severe abuse occurs in our society. Abusing parents often come from families where they had been unhappy or abused. The factors leading to poor quality parenting are of far more concern than the methods used to diagnose these tragedies.

1 Rosen CL, Frost JD, Bricker T, Tarnow JD, Gillette PC, Dunlavy S. Two siblings with recurrent cardiorespiratory arrest: Munchausen syndrome by proxy or child abuse? Pediatrics 1983;71:715-20.

2 Southall DP, Stebbens VA, Rees SV, Lang MH, Warner JO, Shinebourne EA. Apnoeic episodes induced by smothering: two cases identified by covert video surveillance. $B M 7$ 1987;294:1637-41.

3 Esptein MA, Markovitz RL, Gallo DM, Holmes JW, Gryboski JD. Munchausen syndrome by proxy: considerations in diagnosis and confirmation by video syrveillance. Pediatrics 1987;80:220-4.

4 Meadow R. Suffocation, recurrent apnea, and sudden infant death. 7 Pediatr 1990;117:351-7.

5 Alexander $R$, Smith $W$, Stevenson R. Serial Munchausen syndrome by proxy. Pediatrics 1990;86:581-5.

6 Samuels MP, McClaughlin W, Jacobson RR, Poets CF, Southall DP. Fourteen cases of imposed upper airway obstruction. Arch Dis Child 1992;67:162-70.

7 Williams C, Bevan VT. The secret observation of children in hospital. Lancet 1988;: :780-1.

8 Waller DA. Obstacles to the treatment of Munchausen by proxy syndrome. fournal of the American Academy of Child Psychiatry 1983;22:80-5.

(Accepted 9 Augsut 1993) 